\title{
Câncer de Cólon: Como Diagnosticá-lo? Trabalho Prospectivo
}

\author{
Colon Cancer: How can we Diagnose it? a Prospective Study
}

\author{
EDSON JURADO DASILVA ${ }^{1 \mathrm{e} 2} ;$ DANIEL FREIRE $^{1}$; YEDADE SOUZA ${ }^{1} ;$ ELEODOROALMEIDA $^{1}$ \\ Serviço de Coloproctologia do HSE - Ministério da Saúde RJ ${ }^{1}$; Serviço de Endoscopia Digestiva da \\ Casa de Portugal ${ }^{2}$ - Rio de Janeiro - RJ - Brasil.
}

SILVA EJ; FREIRE D; SOUZA Y; ALMEIDA E. Câncer de Cólon: Como Diagnosticá-lo? Trabalho Prospectivo. Rev bras Coloproct, 2007;27(1): 020-025.

RESUMO: Objetivo: Analisar o perfil clínico de portadores de câncer colorretal (CCR). Métodos: Estudo prospectivo com 390 pacientes submetidos a colonoscopia. 152 assintomáticos Grupo A (G-A) e 238 sintomáticos com CCR Grupo B (G-B). Analisamos história familiar (HF), sintomas, Índice de massa Corpórea (IMC), localização do tumor e histopatologia. $P<0,05$ foi considerado significativo. Resultados: Média de idade, 62+/-9 G-A e 61+/-13 G-B, P>0,05. HF positiva em 79 (31\%) com CCR, contra 34 (32\%) sem câncer, P>0,05. IMC no G-A foi 25+/-4 contra 27+/-4 no G-B P>0,05. No G-A 9 (5,9\%) tiveram CCR. Câncer superficial em 1 (11\%) no G-A contra 21 (8,8\%) no G-B, P>0,05. Câncer em cólon esquerdo em 6 (66\%) no G-A contra 168 (69\%) no G-B, P>0,05. Alteração do hábito intestinal com sangue, cólon esquerdo $56(60 \%)$ contra $13(31 \%)$ no direito P<0,01. Emagrecimento com anemia mais freqüentes em tumor de cólon direito quando comparado com esquerdo, $30(44 \%)$ versus $31(17 \%)$ e 36 (46\%) versus $15(8 \%)$ p<0,01. Sintomas há mais de 6 meses em 157 (63\%) Adenocarcinoma moderadamente diferenciado em 166 (67\%), bem diferenciado em $63(25 \%)$ e pouco diferenciado em 18 (7\%). Conclusões: O perfil de risco para CCR precisa ser aprimorado.

Descritores: Câncer colorretal, diagnóstico, colonoscopia, fatores de risco.

\section{INTRODUÇÃO}

Estimativas de incidência de câncer colo-retal (CCR) no Brasil para 2006 publicadas pelo INCA, apontam esta neoplasia como o quinto tumor maligno mais freqüente entre homens (11300 casos novos) e o quarto entre as mulheres (13970 casos novos $\left.{ }^{1}\right)$. Existe forte componente genético, e por mais que nos esforcemos o diagnóstico geralmente é feito em sua fase avançada.

O objetivo deste trabalho é comparar o perfil clínico de portadores de CCR com um grupo de pessoas assintomáticas.

\section{PACIENTES E MÉTODOS}

Estudo prospectivo realizado no período de dezembro de 2003 a novembro de 2005 , constando de
390 pacientes adultos submetidos à colonoscopia. 152 assintomáticos com idade mínima de 50 anos (Grupo A) e 238 portadores de câncer colorretal, com diagnóstico feito na endoscopia (Grupo B). Biópsias, história familiar $(\mathrm{HF})$, sintomas apresentados, Índice de Massa Corpórea (IMC), localização do tumor e histopatologia foram analisados. Teste $t$ de Student e Qui-quadrado foram utilizados para avaliação estatística. $P<0,05$ foi considerado significativo. Portadores de doença inflamatória intestinal e adenomatose familiar foram excluídos do estudo.

\section{RESULTADOS}

Foram incluídos 390 pacientes, sendo 152 assintomáticos (Grupo A) e 238 portadores de CCR (Grupo B). A faixa etária em anos dos respectivos grupos está representada na Tabela 1 .

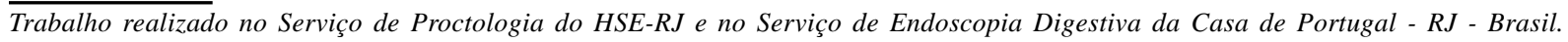

Recebido em 10/12/2006

Aceito para publicação em 08/02/2007 
A História familiar para CCR foi estudada sem a pretensão de se avaliar o grau de parentesco, isto é, apenas positividade e negatividade. Estes dados foram coletados durante consulta prévia ao exame colonoscópico e está representada na Tabela 2 .

Os dados relacionados ao IMC dos grupos estudados se encontram na Tabela 3.

Apesar do Grupo A ser de sem sintomas, que fizeram colonoscopia de rotina, encontramos CCR em $9(5,9 \%)$ sendo superficial em apenas $1(11 \%)$.

A presença de adenoma nos dois grupos estudados se encontra representada na Tabela 4.

Para análise topográfica do CCR optamos pelo estudo da distribuição anatômica em sua forma simplificada em cólon direito CD e cólon esquerdo CE (2), estando representada na Tabela 5.

Tabela 1 - Idade dos grupos.

\begin{tabular}{lccc}
\hline & G-A & G-B & P \\
\cline { 2 - 4 } Idade & $62+/-9$ & $61+/-13$ & $>0,05$ \\
\hline
\end{tabular}

Tabela 2 - História familiar para CCR.

\begin{tabular}{lll}
\hline & \multicolumn{2}{c}{ HF positiva para CCR } \\
\cline { 2 - 3 } & $\mathbf{N}$ & \% \\
\hline Portadores de CCR & 79 & 31 \\
Pessoas sem CCR & 34 & 23 \\
P $>0,05$ & & \\
\hline
\end{tabular}

Tabela 3 - IMC.

\begin{tabular}{lc}
\hline & IMC \\
\hline G-A & $26+/-4$ \\
G-B & $27+/-4$ \\
P $>0,05$ & \\
\hline
\end{tabular}

Tabela 4 - Adenomas no cólon.

\begin{tabular}{lllll}
\hline & \multicolumn{2}{c}{ G-A } & \multicolumn{2}{c}{ G-B } \\
\cline { 2 - 5 } & $\mathbf{N}$ & $\mathbf{\%}$ & $\mathbf{N}$ & \% \\
\hline $\begin{array}{l}\text { Adenoma } \\
\text { P } 0,05\end{array}$ & 45 & 29 & 61 & 25 \\
\hline
\end{tabular}

Com relação ao estadiamento local, dividimos nossos achados em câncer superficial, aquele que invade até a primeira camada da submucosa, SM1 e câncer avançado, que por ter penetração maior não se presta a tratamento endoscópico. A distribuição da frequiência nos dois grupos analisados se encontra na Tabela 6 .

Lesões sincrônicas malignas só foram detectadas no Grupo B e estão representadas na Tabela 7.

O quadro clínico básico foi estudado também de forma simplificada, dando-se ênfase aos sintomas e/ou sinais principais, confrontando-os com a topografia encontrada. Estes achados podem ser encontrados na Tabela 8.

Outros aspectos do quadro clínico do CCR poderão ser encontrados na Tabela 9.

Nos portadores de CCR o tempo da sintomatologia poderá ser encontrado na Tabela 10.

O diagnóstico histopatológico encontrado corresponde a Tabela 11 .

Tabela 5 - Distribuição anatômica do CCR nos dois grupos estudados.

\begin{tabular}{lcccc}
\hline & \multicolumn{2}{c}{ G-A } & \multicolumn{2}{c}{ G-B } \\
\cline { 2 - 5 } & $\mathbf{N}$ & $\boldsymbol{\%}$ & $\mathbf{N}$ & $\boldsymbol{\%}$ \\
\hline $\mathrm{CE}$ & 6 & 66 & 168 & 69 \\
$\mathrm{CD}$ & 3 & 33 & 70 & 30 \\
$\mathrm{P}>0,05$ & & & & \\
\hline
\end{tabular}

Tabela 6 - Estadiamento local.

\begin{tabular}{lrrrr}
\hline & \multicolumn{2}{c}{ G-A } & \multicolumn{2}{c}{ G-B } \\
\cline { 2 - 5 } & $\mathbf{N}$ & $\mathbf{\%}$ & \multicolumn{1}{c}{$\mathbf{N}$} & \multicolumn{1}{c}{$\%$} \\
\hline Câncer superficial & 1 & 11 & 21 & 8,8 \\
Câncer avançado & 8 & 89 & 217 & 91,1 \\
Total & & 9 & & 238 \\
P $>0,05$ & & & & \\
\hline
\end{tabular}

Tabela 7 - Lesões sincrônicas malignas.

\begin{tabular}{lll}
\hline & \multicolumn{2}{c}{ G-B } \\
\cline { 2 - 3 } & $\mathbf{N}$ & \% \\
\hline Lesões sincrônicas malignas & 6 & 2,4 \\
\hline
\end{tabular}


Tabela 8 - Quadro clínico relacionado à topografia do CCR.

\begin{tabular}{|c|c|c|c|c|c|}
\hline & \multicolumn{2}{|c|}{$\mathbf{C E}$} & \multicolumn{2}{|c|}{ CD } & \multirow[t]{2}{*}{$\mathbf{P}$} \\
\hline & $\mathbf{N}$ & $\%$ & $\mathbf{N}$ & $\%$ & \\
\hline Alteração dos hábitos intestinal com sangue & 56 & 60 & 13 & 31 & $<0,01$ \\
\hline Perda de peso acentuada & 31 & 17 & 30 & 44 & $<0,01$ \\
\hline Anemia importante & 15 & 8 & 36 & 46 & $<0,01$ \\
\hline
\end{tabular}

Tabela 9 - CCR Outros aspectos.

\begin{tabular}{lrc}
\hline & \multicolumn{2}{c}{ CCR } \\
\cline { 2 - 3 } & \multicolumn{1}{c}{ N } & \% \\
\hline Obstrução intestinal aguda & 21 & 8,5 \\
Síndrome de Fournier & 2 & 0,8 \\
Quadro semelhante apendicite & 1 & 0,4 \\
\hline
\end{tabular}

Tabela 10 - Tempo de sintomas nos portadores de CCR.

\begin{tabular}{lrr}
\hline & N & \% \\
\hline Sintomas presentes há mais de 6 meses & 157 & 63,5 \\
Sintomas presentes há menos de 6 meses & 90 & 36,4 \\
\hline
\end{tabular}

\section{DISCUSSÃO}

Nosso estudo teve por base um grupo de pessoas assintomáticas, e pacientes com sintomas que não se enquadravam nos riscos convencionais para o diagnóstico do CCR.

A obesidade e o IMC costumam ser identificados como fatores de risco para o desenvolvimento do CCR, embora os achados nem sempre sejam consistentes ${ }^{3,4,5,6,7}$. Na nossa casuística este aspecto não foi relevante, tabela-3. É possível que a relação cintura/ quadril, refletindo melhor a obesidade abdominal tenha maior impacto na gênese do CCR ${ }^{8}$. Fator adici- onal que poderia estar influenciando nossos resultados, seria a inclusão de câncer de reto no nosso estudo, pois parece que este tumor não estaria associado a dados antropométricos ${ }^{9}$.

A história familiar também não diferiu nos 2 grupos por nós analisados tabela 2, provavelmente por não termos incluído grupos de risco convencionais como por exemplo; adenomatose familiar e síndrome de Lynch de acordo com os critérios mais recentes ${ }^{10}$ e por termos computado tumor de reto juntamente com o tumor de cólon ${ }^{11,12}$.

Além do quadro clínico, a seleção para indicação de colonoscopia deverá incluir dados referentes à história familiar, embora a confirmação de síndrome de Lynch ou de câncer de cólon hereditário não associado a polipose (HNPCCC) deverá ser diagnosticada preferencialmente em base molecular e na presença da instabilidade microssatélite (MSI) ${ }^{13,14,15,16}$, que não fizeram parte de nossa avaliação.

A presença de adenoma teve freqüência semelhante nos dois grupos estudados, como se pode ver na Tabela 4, refletindo a grande prevalência desta lesão, precursora de neoplasia maligna do cólon ${ }^{17,18,19}$. Nosso grupo de assintomáticos com idade acima de 50 anos teve a taxa de $29 \%$ de adenoma, semelhante à encontrada por outros autores que foi de $21,3 \%$ (20). Lesões sincrônicas malignas no cólon ocorreram somente nos portadores de CCR sintomáticos, correspondendo à alíquota de $2,4 \%$, que pode ser vista na Tabela-7 e é semelhante à encontrada por outros autores, variando de 2,12 a $4 \%{ }^{21,22,23,24}$.

Tabela 11 - Diagnóstico histopatológico do CCR.

\begin{tabular}{lrr}
\hline & N & \% \\
\hline Adenocarcinoma moderadamente diferenciado & 166 & 67 \\
Adenocarcinoma bem diferenciado & 63 & 25 \\
Adenocarcinoma mucinoso e pouco diferenciado & 18 & 7 \\
\hline
\end{tabular}


A nossa distribuição topográfica do CCR seguiu o padrão habitual para população de risco médio tanto no Grupo A como no grupo B, como podemos ver na Tabela-5 predominando portanto em cólon esquerdo, incluindo reto, correspondendo a 66 e $69 \%$ respectivamente e diferente do padrão inverso alcançado na HNPCC e ou na síndrome de Lynch, como se observa na literatura 25, 26, 27, 28, 29, 30, 31, 32, 33, 34, 35, 36, 37 .

Nos dois grupos estudados houve total predomínio do câncer avançado sobre o superficial como podemos observar na Tabela 6 , e nos sintomáticos o tempo de sintomas foi inferior a 6 meses em quase $40 \%$ deste grupo, como se vê na Tabela 10 . Ambos os achados também estão presentes na literatura ${ }^{38}$.

O diagnóstico histopatológico se encontra na Tabela 11 refletindo o perfil de pacientes de grupo risco médio para CCR e está de acordo com a literatura $39,40,41$.

\section{CONCLUSÕES}

1. O câncer colorretal é freqüentemente diagnosticado em sua fase avançada, inclusive em pessoas assintomáticas submetidas a rastreamento por colonoscopia.

2. No grupo de risco médio para esta doença, a história familiar e o índice de massa corpórea tiveram pouca importância no diagnóstico.

3. Cerca de $40 \%$ dos pacientes portadores de câncer colorretal tiveram sintomas com menos de 6 meses de evolução, apesar do estágio avançado da doença.

4. Precisamos melhorar nossos métodos para o diagnostico de câncer colorretal, desenvolvendo perfil de risco, que permita a seleção apropriada para indicar colonoscopia atendendo a população de risco médio, com vistas a diagnóstico mais precoce.

\begin{abstract}
Purpose: Analyze the profile of colon and rectal cancer. Methods: 390 colonoscopies were performed in 152 asymptomatic people Group A and 238 colon cancer patients with diagnosis done at colonoscopy Group B. Age, family history (FH), symptoms, Body Mass Index (BMI), location of the tumor and histopathology were analyzed. $P$ value $<0,05$ was significant. Results: Mean age Group A 62+/-9 years and 61+/-13 Group B P>0,95. 79 patients (31\%) of cancer had positive FH and positive FH was present in 34 patients without cancer (23\%) P>0,05.BMI was 26+/-4 Group A and 27+/- for B P>0,05 9 patients (5,9\%) from Group A was found to have cancer. Superficial in 1 (11\%) Group A against $21(8,8 \%)$ for Group B P>0,05. Change of bowel habits with bleeding was $56(60 \%)$ on the left colon cancer against $13(31 \%)$ on the right $P<0,01$. Weight loss and anemia occurred more frequently on the right when compared to the left $30(44 \%)$ X $31(17 \%)$ and $36(46 \%) \times 15(8 \%) P<0,01.157$ patients $(63 \%)$ had symptoms for more than 6 months. Moderated-differentiated adenocarcinoma was found in $166(67 \%)$, well-differentiated 63 $(25 \%)$ and poorly-differentiated in $18(7 \%)$ of the patients. Conclusion: The profile of CRC must be better studied.
\end{abstract}

Key words: Colon, cancer, colonoscopy, diagnose.

\section{REFERÊNCIAS}

1. Ministério da Saúde INCA www.inca.gov.br Documento obtido via on line em 24/09/2006.

2. Gómez D, Dalal Z, Raw E, Roberts C, Lyndon PJ. Anatomical distribution of cancer over a 10 year period in a district general hospital: is there a true rightward shift? Postgrad med J 2004; 80:667-9.

3. Calle EE, Thun MJ. Obesity and cancer Oncogene.2004; 23:6365-78.

4. Gunter MJ, Leitzmann MF. Obesity and colorectal cancer:epidemiology, mechanisms and candidate gens. J Nutr Biochem 2006; 17:145-56.

5. Lukanova A, Bjor O, Kaaks R, Lenner P, Lindahl B, Hallmans G et al. Body mass index and cancer: results from the Northern Sweden health and disease Cohort. Int J Cancer 2006; 118:458-66.
6. Rapp K, Schroeder J, Klenk J, Stoehr S, Ulmer H, Concin H et al. Obesity and incidence of cancer: a large cohort study of over 145000 adults in Austria. Br J cancer2005;93:1062-7.

7. Rousseau MC, Parent ME, Siemiatycki J. Comparison of self-reported height and weight by cancer type among men from Montreal, Canada. Eur J Cancer Prev 2005;14:431-8.

8. Frezza EE, Wachtel MS, Chiriva-Internati M. Influence of obesity on risk of developing colon cancer. Gut 2006; 55:285-91.

9. Pischon T, Lahmann PH, Boeing H, Friedenreich C, Norat T, Tjonneland A et al. Body size and risk of colon and rectal cancer in the European Prospective Investigation Into Cancer and Nutrition (EPIC). J Natl Cancer Inst2006;98:920-31.

10. Vasen HFA, Watson P, Mecklin JK, Lynch HT. New Clinical Criteria for Hereditary Nonpolyposis Colorectal Cancer HNPCC, Lynch Syndrome - proposed by the International Collaborative Group on HNPCC. Gastroenterology1999; 116:1453-56. 
11. Andrieu N, Launoy G, Guillois R, Ory-Paoletti C, Gignoux $M$. Estimation of the familial relative risk of cancer by site from a French population based family study on colorectal cancer ( CCREF study ). Gut2004; 53:1322-8.

12. Stefansson T, Moller PH, Sigurdsson F, Steingrimsson E, Eldon BJ. Familial risk of colon and rectal cancer in Iceland: evidence for different etiologic factors? Int J cancer2006; 119:304-8.

13. Dove-Edwin I, de Jong AE, Adams J, Mesher D, Lipton L, Sasieni P et al. Prospective results of surveillance colonoscopy in dominant familial colorectal cancer with and without Lynch syndrome. Gastroenterology 2006; 130:1995-0.

14. Kerber RA, Neklason DW, Samowitz WS, Burt RW. Frequency of familial colon cancer and hereditary nonpolyposis colorectal cancer (Lynch syndrome ) in a large population database. Fam Cancer 2005; 4:239-44.

15. Lipton LR, Johnson V, Cummings C, Fisher S, Risby P, Eftekhar Sadat AT et al. Refining the Amsterdam Criteria and Bethesda Guidelines: testing algorithms for the prediction of mismatch repair mutation status in the familial cancer clinic. J Clin Oncol 2004; 22:4934-43.

16. Muller A, Edmonston TB, Dietmaier W, Buttner R, Fishel R, Ruschoff J. MSI-testing in hereditary non-polyposis colorectal carcinoma ( HNPCC ). Dis Markers 2004; 20:225-36.

17. Lindgren G, Liljegren A, Jaramillo E, Rubio C, Lindblom A. Adenoma prevalence and cancer risk in familial non-polyposis colorectal cancer. Gut2002; 50:228-34.

18. Ponz de Leon M, Di Gregorio O. Pathology of colorectal cancer. Dig Liver Dis 2001;33:372-88.

19. Reinacher-Schick A, Schmiegel W. Surveillance strategies in patients after polypectomy. Dig Dis 2002;20:61-9.

20. Strul H, Kariv R, Leshno M, Halak A, Jakubowicz, Santo M et al. The prevalence rate and anatomic location of colorectal adenoma and cancer detected by colonoscopy in average-risk individuals aged 40-80 years. Am J Gastroenterolo 2006;101:263-5.

21. Arenas RB, Fichera A, Mhoon D, Michelassi F. Incidence and therapeutic implications of synchronous colonic pathology in colorectal adenocarcinoma. Surgery 1997;122:709-10.

22. Nikoloudis N, Saliangas K, Economou A, Andreadis E, Siminou S, Manna I et al. Synchronous colorectal cancer. Tech Coloproctol 2004;8 Suppl1:s177-9.

23. Piñol V, Andreu M, Castells A, Payá A, Bessa X, Jover R. Synchronous Colorectal Neoplasms in Patients with Colorectal Cancer: Predisposing Individual and Familial Factors. Dis Colon Rectum 2004;47:1192-00.

24. Takeuchi H, Toda T, Nagasaki S, Kawano T, Minamisono Y, Maehara $\mathrm{Y}$ et al.Synchronous multiple colorectal adenocarcinomas. J Surg Oncol 1997; 64:304-7.

25. Benatti P, Roncucci L, Ganazzi D, Percesepe A, Di Gregorio $\mathrm{C}$, Pedroni $\mathrm{M}$ et al.Clinical and biologic heterogeneity of hereditary nonpolyposis colorectal cancer. Int J Cancer 2001;95:323-8.
26. Cai SJ, Xu Y, CaiGX, Lian P, Guan ZQ, Mo SJ et al. Clinical characteristics and diagnosis of patients with hereditary nonpolyposis colorectal cancer. World J Gastroenterol 2003;9:284-7.

27. Chai SM, Zeps N, Shearwood AM, Grieu F, Charles A, Harvey $\mathrm{J}$ et al. Screening for defective DNA mismatch repair in stage II and III colorectal cancer patients. Clin Gastroenterol hepatol 2004;2:1017-25.

28. Corleto VD, Zykaj E, Mercantini P, Pilozzi E, Rossi M, Carnuccio A et al.Is colonoscopy sufficient for colorectal cancer surveillance in all HNPCC patients? World J Gastroenterol 2005;11:7541-4.

29. Hemminki K, Li X. Familial colorectal adenocarcinoma and hereditary nonpolyposis colorectal cancer: a nationwide epidemiological study from Sweden. Br J cancer 2001;84:96974.

30. Iacopetta BG. Are there two sides to colorectal cancer? Int $\mathbf{J}$ Cancer 2002;101:403-8.

31. Kouraklis G, Misiakos EP. Hereditary nonpolyposis colorectal cancer (Lynch syndrome): criteria for identification and management. Dig Dis Sci 2005;50:336-44.

32. Lin KM, Shashidharan M, Tement CA, Thorson AG, Blatchford GJ, Christensen MA et al. Colorectal and extracolonic cancer variations in MLH1/MSH2 hereditary nonpolyposis colorectal cancer kindred and the genral population. Dis Colon Rectum1998;41:428-33.

33. Lynch HT, Lynch JF. Hereditary nonpolyposis colorectal cancer. Semin Surg Oncol 2000;18:305-13.

34. Michalopoulos NV, Saetta AA, Lazaris AC, Gigelou F, Koilakou S, Patsouris E et al. Microsatellite instability in sporadic and inherited colon adenocarcinomas from Greek patients; correlation with several clinicopathological characteristics. Acta Gastroenterol Belg 2005;68:294-1.

35. Rijcken FE, Hollema H, kleibeuker JH. Proximal adenomas in hereditary non-polyposis colorectal cancer are prone to rapid malignant transformation. Gut 2002;50:382-6.

36. Soreide K, Janssen EA, Soilland H, Komer H, Baak JP. Microsatellite instability in colorectal cancer. Br J surg 2006;93:395-6.

37. Wong VK, Yoshida EM, Ryan Ag, Ho SG, Salh B. Advanced colon cancer before the age of 20 years: a case for extension of current colonoscopy surveillance guidelines in hereditary nonpolyposis colorectal cancer syndrome. Can J Gastroenterol 2005; 18:319-20.

38. Schoepfer A, Marbet UA. Colonoscopic findings of symptomatic patients aged 50 to 80 years suggest that workup of tumor suspicious symptoms hardly reduces cancerinduced mortality. Swiss Med Wkly 2005; 135:679-83.

39. Kang H, O`Connell JB, Maggard MA, Sack J, Ko CY. A 10year outcomes evaluation of mucinous and signet-ring cell carcinoma of the colon and rectum. Dis Colon Rectum 2005; 48:1116-8. 
40. Luo DC, Cai Q, Sun MH, Ni SC, Chen ZJ, Li XY et al. Clinicopathological and molecular genetic analysis of HNPCC in China. World J Gastroenterol 2005; 11:1673-9.

41. Ruschoff J, Dietmaier W, Bocker T, Wallinger S, Kullmann F, Beham A et al. Molecular cancer disposition diagnosis exemplified by colorectal carcinoma. What is the contribution of pathology? Pathologe 1998;19:269-78.
Endereço para correspondência: EDSON JURADO DA SILVA

Rua Rodolfo de Souza 105 - Vila Isabel

Rio de Janeiro, RJ

20551-270

TEL/FAX (21) 2520-5115

e-mail edsonjurado@alternex.com.br 